Check for updates

Cite this: J. Mater. Chem. B, 2019, 7, 5983

Received 16th June 2019, Accepted 2nd September 2019

DOI: $10.1039 / c 9 t b 01202 h$

rsc.li/materials-b

\section{A pH-sensitive polymer based precise tumor targeting strategy with reduced uptake of nanoparticles by non-cancerous cells $\dagger$}

\author{
Zihou Li, ${ }^{\text {abcd }}$ Xuehua Ma, ${ }^{a}$ Yuanzhi Xia, ${ }^{a}$ Kun Qian, ${ }^{\text {ad }}$ \\ Ozioma Udochukwu Akakuru, (D) ${ }^{a}$ Lijia Luo, ${ }^{\text {ad }}$ Jianjun Zheng, ${ }^{e}$ Ping Cui, ${ }^{a}$ \\ Zheyu Shen (D)*a and Aiguo Wu (D)*a
}

\begin{abstract}
Drug-loaded nanoparticles can be specifically uptaken by tumor cells to realize active targeting due to the conjugated ligands or antibodies on their surface. However, some non-cancerous cells express non-specific receptors or antigens on their surface, which can react with the ligands or antibodies conjugated on the nanoparticle surface and then result in non-specific uptake of the nanoparticles by non-cancerous cells. In order to reduce the non-specific uptake of nanoparticles by non-cancerous cells, in this study, we proposed a $\mathrm{pH}$-sensitive polymer based precise tumor targeting strategy and synthesized superparamagnetic iron oxide nanoparticle (SPION) encapsulated albumin nanoparticles (AN) with conjugation of folic acid (FA) and mPEG-DCA (SPION-AN-FA(amPEG), in which mPEG can shield FA, avoiding the non-specific recognition by normal cells under physiological conditions, and can be shed to expose FA in tumor microenvironments. The $\mathrm{pH}$-sensitivity of $\mathrm{mPEG}-\mathrm{DCA}$ was verified by HPLC characterization and ${ }^{1} \mathrm{H}-\mathrm{NMR}$ spectroscopy. The graft density and length of mPEG-DCA were optimized via the cellular uptake of SPION-AN-FA@mPEG measured by flow cytometry analysis. The $r_{2}$ value and $r_{2} / r_{1}$ ratio of the optimized SPION-AN-FA@MPEG (i.e., SPION-AN-FA(amPEG4) are $168.6 \mathrm{mM}^{-1} \mathrm{~s}^{-1}$ and 42.8, respectively, which are both much higher than that of the commercial contrast agent Resovist ${ }^{\circledR}$. The in vitro $T_{2}$-weighted $M R$ images and in vivo MRI performance demonstrate that our SPION-AN-FA@mPEG4 nanoparticles can be used as an effective $T_{2}$-weighted MRI contrast agent.
\end{abstract}

\section{Introduction}

Conventional therapies including chemotherapy, ${ }^{1,2}$ radiotherapy ${ }^{3,4}$ and surgery ${ }^{5,6}$ are important modalities for cancer treatment. However, surgery is not applicable to tumors at late stages. Both chemotherapy and radiotherapy have serious side effects

\footnotetext{
${ }^{a}$ Cixi Institute of Biomedical Engineering, CAS Key Laboratory of Magnetic Materials and Devices, \& Key Laboratory of Additive Manufacturing Materials of Zhejiang Province, Ningbo Institute of Materials Technology and Engineering, Chinese Academy of Sciences, Ning-bo, Zhejiang 315201, China.

E-mail: shenzheyu@nimte.ac.cn, aiguo@nimte.ac.cn; Tel: +8657487617278, +8657486685163

${ }^{b}$ School of Physical Science and Technology, ShanghaiTech University, Shanghai 200031, China

${ }^{c}$ Shanghai Institute of Ceramics, Chinese Academy of Sciences, Shanghai 200050, China

${ }^{d}$ Institute of Physics, University of Chinese Academy of Sciences, Beijing 100190, China

${ }^{e}$ HuaMei Hospital, University of Chinese Academy of Sciences, 41 Northwest Street, Ning-bo, Zhejiang, 315010, China

$\dagger$ Electronic supplementary information (ESI) available. See DOI: 10.1039/c9tb01202h
}

on normal tissues and cells, which cause great pain to patients. ${ }^{7,8}$ In the past few decades, to overcome the side effects of chemotherapy, drug delivery systems have been widely developed to load chemotherapeutic agents for tumor-specific delivery (i.e., tumor targeting). The tumor targeting mainly includes passive and active targeting. ${ }^{9,10}$

For passive targeting, the drug delivery systems (i.e., drugloaded nanoparticles) circulate in the blood stream and accumulate in tumor sites due to the enhanced permeability and retention (EPR) effect. For example, Moitra and co-workers developed pH-responsive gemini lipid derived co-liposomes for efficacious doxorubicin (DOX) delivery to DOX-resistant HeLa cancer cells. ${ }^{11}$ Zhang et al. synthesized a multifunctional hybrid liposome to embed indocyanine green and a magnetic resonance imaging (MRI) contrast agent as a theranostic platform for MRI-guided photothermal therapy (PTT). ${ }^{12}$

For active targeting, the drug-loaded nanoparticles can be specifically uptaken by tumor cells due to the conjugated targeting molecules on their surface, e.g., ligands, ${ }^{13,14}$ antibodies, ${ }^{15-18}$ peptides $^{19,20}$ or aptamers. ${ }^{21,22}$ For example, Ye et al. 
conjugated galactose ligands onto an integrated therapeutic delivery system to realize an enhanced treatment of hepatocellular carcinoma. ${ }^{23}$ Zhang et al. grafted folic acid (FA) onto the surface of graphene quantum dots to selectively target cancer cells with different levels of folate receptors. ${ }^{24}$ However, some non-cancerous cells express non-specific receptors or antigens on their surface, which can react with the ligands or antibodies conjugated on the nanoparticle surface and then result in non-specific uptake of the nanoparticles by non-cancerous cells.

In order to reduce the non-specific uptake of nanoparticles by non-cancerous cells, in our previous study, we proposed a Trojan-horse targeting strategy. ${ }^{25}$ Typically, FA and a $\mathrm{pH}$-sensitive polymer (PP) were conjugated onto the surface of albumin nanoparticles (AN). The PP can hide FA (small molecule) at pH 7.4 and shrink to expose FA at pH 5.5, resulting in reduced uptake of the nanoparticles by normal healthy cells with non-specific receptors. However, this strategy still has a problem that some of the shrunk PP can cover the FA molecules on the surface of the nanoparticles resulting in a decreased efficiency of FA targeting.

In this study, we propose a new $\mathrm{pH}$-sensitive polymer based precise tumor targeting strategy with reduced uptake of nanoparticles by non-cancerous cells (Fig. 1). Briefly, superparamagnetic iron oxide nanoparticles (SPION) were encapsulated in AN to construct the SPION-AN nanoparticles. Then, FA and mPEG were conjugated onto the surface of SPION-AN to synthesize the SPION-AN-FA@mPEG nanoparticles (Fig. 2). As shown in Fig. 1, SPION-AN-FA@mPEG nanoparticles have a long circulation time in blood and cannot be recognized by normal healthy cells without receptors or those with nonspecific receptors because the targeted molecule FA is hidden in mPEG. However, in tumor microenvironments, the mPEG can be shed, and the FA can be exposed to be recognized by the cancer cells with folate receptor $\alpha(\mathrm{FR} \alpha)$. The nanoparticles can then be internalized in the cancer cells due to the FR $\alpha$-mediated endocytosis. Therefore, our SPION-AN-FA@mPEG can be used as a MRI contrast agent with precise tumor targeting.

\section{Materials and methods}

\subsection{Preparation of SPION}

A polyol method was used to prepare the superparamagnetic iron oxide nanoparticles (SPION). ${ }^{26}$ Typically, $2.0 \mathrm{~mL}$ of an aqueous solution of $\mathrm{FeCl}_{3} \cdot 6 \mathrm{H}_{2} \mathrm{O}(202.7 \mathrm{mg})$ and $\mathrm{FeCl}_{2} \cdot 4 \mathrm{H}_{2} \mathrm{O}$ (178.9 mg) was added into $100 \mathrm{~mL}$ of diethylene glycol (DEG) solution. After refluxing at $170{ }^{\circ} \mathrm{C}$ for $15 \mathrm{~min}, 5.0 \mathrm{~mL}$ of $\mathrm{NaOH}$ solution $(3.0 \mathrm{M})$ was quickly added into the mixture. After $1 \mathrm{~h}$, the mixture was cooled to room temperature to obtain SPION. The obtained SPION was then washed with $1.0 \mathrm{M} \mathrm{HNO}_{3}$ solution and ethanol 5 times and collected by a neodymium magnet.

\subsection{Preparation of SPION-AN-FA nanoparticles}

Purified SPION (4.4 mg mL $\mathrm{mL}^{-1}, 0.5 \mathrm{~mL}$ ) was added into bovine serum albumin (BSA) aqueous solution (2.0 mL, $40.0 \mathrm{mg} \mathrm{mL}^{-1}$ ) under stirring with an impeller for $1 \mathrm{~h}$. After that, $12.0 \mathrm{~mL}$ of ethanol was added dropwise $\left(2.3 \mathrm{~mL} \mathrm{~min}^{-1}\right)$ into the mixture. When the ethanol was running out, $120.0 \mu \mathrm{L}$ of glutaraldehyde aqueous solution ( $8.0 \mathrm{wt} \%$ ) was rapidly added to crosslink the obtained nanoparticles. $24 \mathrm{~h}$ later, $2.0 \mathrm{~mL}$ of lysine solution (40.0 $\mathrm{mg} \mathrm{mL}^{-1}$ ) was added to react with the free aldehyde groups. After stirring for another $2 \mathrm{~h}$, SPION-AN was centrifuged $(18000 \mathrm{~g}, 10 \mathrm{~min})$ and washed thrice with Milli-Q water.

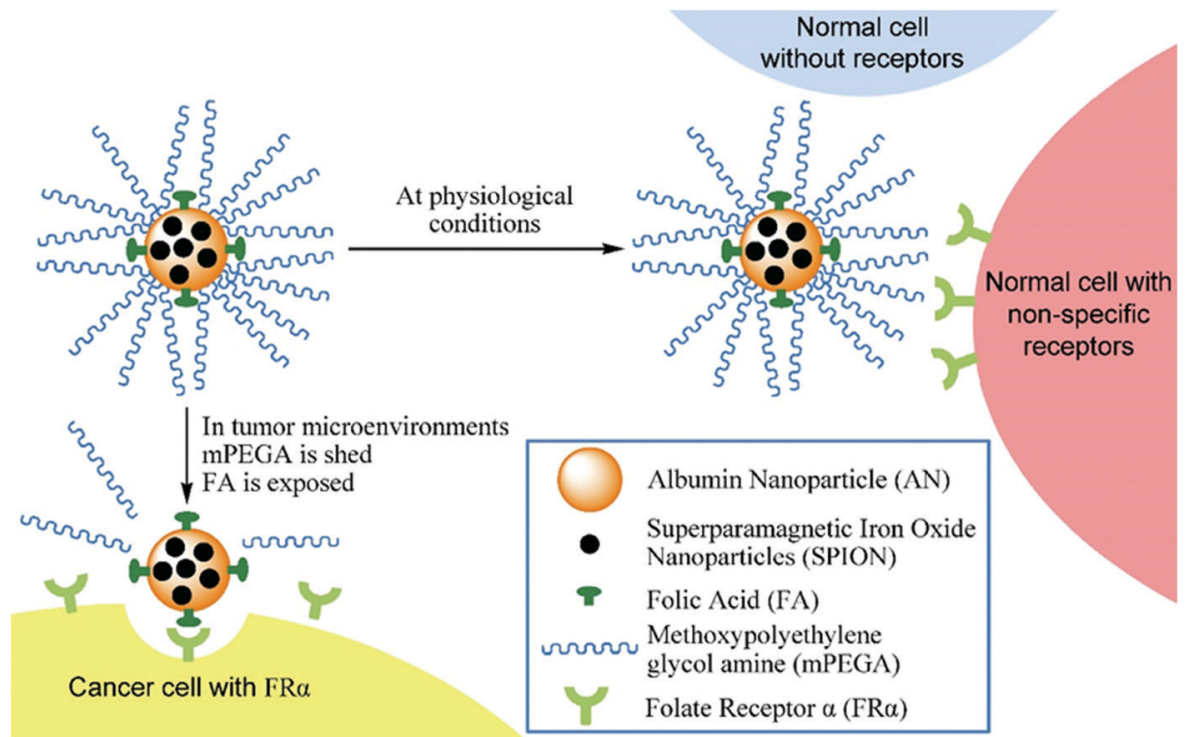

Fig. 1 Design of the precisely targeted $T_{2}$-weighted MRI contrast agent and the mechanism of diminishing non-specific uptake by normal healthy cells. Because the targeted molecule FA is hidden in mPEG, SPION-AN-FA@mPEG nanoparticles have a long circulation time in blood and cannot be recognized by normal healthy cells without receptors or those with non-specific receptors. However, in tumor microenvironments, the mPEG is shed, and the FA is exposed to be recognized by the cancer cells with FR $\alpha$. 

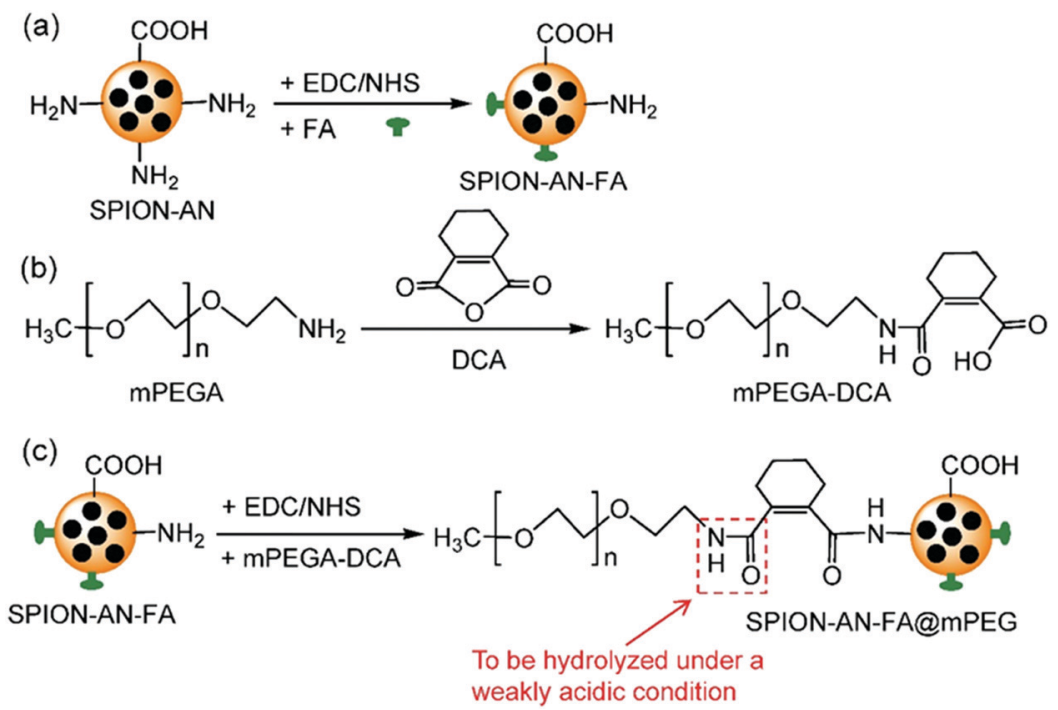

Fig. 2 Synthesis procedures of the precisely targeted $T_{2}$-weighted MRI contrast agent, i.e., SPION-AN-FA@mPEG nanoparticles. The linker between AN and $\mathrm{mPEG}$, i.e., a special amide bond, is stable under normal physiological conditions, but it can be hydrolyzed under a weakly acidic condition resulting in shedding of $\mathrm{MPEG}$ from the nanoparticles.

The purified SPION-AN was subsequently dispersed in pure water for further use. An aliquot of the SPION-AN dispersion was dried at $70{ }^{\circ} \mathrm{C}$ to calculate its concentration.

The reaction between- $\mathrm{COOH}$ of $\mathrm{FA}$ and $-\mathrm{NH}_{2}$ of $\mathrm{AN}$ was utilized to conjugate FA onto the surface of SPION-AN. Briefly, $20 \mathrm{mg}$ of 1-ethyl-3-(3-dimethylaminopropyl) carbodiimide hydrochloride (EDC. $\mathrm{HCl}$ ) and $12.0 \mathrm{mg}$ of $N$-hydroxysuccinimide (NHS) were added into $25 \mathrm{~mL}$ of ice-cooled FA solution $\left(1.0 \mathrm{mg} \mathrm{mL} \mathrm{m}^{-1}\right.$ in PBS, $\left.\mathrm{pH} \sim 7.4\right)$ and stirred for $12 \mathrm{~h}$ at room temperature to obtain activated FA (i.e., FA-NHS). After that, $0.3 \mathrm{~mL}$ of the solution was added into $10 \mathrm{~mL}$ of $1 \mathrm{mg} \mathrm{mL} \mathrm{m}^{-1}$ SPION-AN solution in pure water, and the mixture was stirred at room temperature for $12 \mathrm{~h}$. The resulting FA-conjugated SPION-AN (SPION-AN-FA) dispersion was then centrifuged (18000 g, $10 \mathrm{~min}$ ) and washed twice with Milli-Q water. The supernatant was stored for further analysis.

\subsection{Synthesis of $\mathrm{pH}$-sensitive polymers}

$1.0 \mathrm{~g}$ of $\mathrm{mPEG}-\mathrm{NH}_{2}$ (mPEGA) and $82.0 \mathrm{mg}$ of 3,4,5,6-tetrahydrophthalic anhydride (DCA) were dissolved in $10 \mathrm{~mL}$ of Milli-Q water under magnetic stirring. The $\mathrm{pH}$ of the solution was maintained at 8.0-8.5 (monitored by a $\mathrm{pH}$ meter with addition of $1.0 \mathrm{M} \mathrm{NaOH}$ solution on demand). After $12 \mathrm{~h}$, the mixture was purified by centrifugal filtration $(4000 \mathrm{~g})$ for $30 \mathrm{~min}$ with a cut-off of $3 \mathrm{KDa}$ to obtain the mPEGA-DCA polymers. An aliquot of the polymer solution was dried at $70{ }^{\circ} \mathrm{C}$ to calculate the concentration. EDC. $\mathrm{HCl}(150 \mathrm{mg})$ and $\mathrm{NHS}$ (100 mg) were then dissolved in $8 \mathrm{~mL}$ of $80 \mathrm{mg} \mathrm{mL}$ mPEGA-DCA solution with magnetic stirring for $10 \mathrm{~h}$ at room temperature. The $\mathrm{pH}$ of the solution was also monitored and maintained at $\sim 7.4-8.0$ with addition of $0.1 \mathrm{M} \mathrm{NaOH} / \mathrm{HCl}$ solution on demand. The obtained polymer with activated -COOH (i.e., mPEGA-DCA-NHS) was then purified by centrifugal filtration $(4000 \mathrm{~g})$ for $20 \mathrm{~min}$ with a cut-off of $3 \mathrm{KDa}$.
An aliquot of the polymer solution was dried at $70{ }^{\circ} \mathrm{C}$ and the concentration was also calculated.

\subsection{Conjugation of the $\mathrm{pH}$ sensitive polymer on SPION-AN-FA}

The pH-sensitive polymers were grafted onto the surface of SPION-AN-FA by the reaction between $-\mathrm{NH}_{2}$ of $\mathrm{AN}$ and -COOH of polymers. Typically, $0.5 \mathrm{~mL}$ of mPEGA-DCA-NHS solution (molecular weight $=2,5$, and $10 \mathrm{KDa}$ ) and $10.0 \mathrm{~mL}$ of $1.0 \mathrm{mg} \mathrm{mL}{ }^{-1}$ SPION-AN-FA were mixed, respectively. The reaction was continued for $16 \mathrm{~h}$ at room temperature and the $\mathrm{pH}$ was monitored and maintained at $\sim 7.4$ with addition of $0.1 \mathrm{M} \mathrm{NaOH} / \mathrm{HCl}$ solution on demand. The un-reacted polymer was removed by washing and centrifugation $(18000 \mathrm{~g}$, $10 \mathrm{~min})$. The harvested mPEG-conjugated SPION-AN-FA samples (SPION-AN-FA@mPEG) were dispersed in $5 \mathrm{~mL}$ of Milli-Q water. An aliquot of the polymer solution was dried at $70{ }^{\circ} \mathrm{C}$ to calculate the concentration.

\subsection{Characterization of the nanoparticles and polymers}

2.5.1 Determination of SPION loading content, loading efficiency and FA grafting content. The loading content of SPION in SPION-AN-FA was measured by inductively coupled plasma optical emission spectrometry (ICP-OES) using an Optima 2100 DV instrument (PerkinElmer, USA). Typically, $4.0 \mathrm{~mL}$ of $\mathrm{HNO}_{3}(\sim 65-68 \%), 0.5 \mathrm{~mL}$ of $\mathrm{H}_{2} \mathrm{O}_{2}$, and $0.5 \mathrm{~mL}$ of $\mathrm{HClO}_{4}$ were added into $200 \mu \mathrm{L}$ of SPION-AN-FA solution $\left(1.0 \mathrm{mg} \mathrm{mL}^{-1}\right)$ in a reaction kettle. Then, the reaction kettle was placed in an oven at $180{ }^{\circ} \mathrm{C}$. After $4 \mathrm{~h}$, the decomposition reaction was finished and the sample was cooled down to room temperature. Then, the sample was diluted to $10 \mathrm{~mL}$ with Milli-Q water and measured with ICP-OES. A calibration curve was constructed via ICP-OES measurement of iron standard solutions with different concentrations (containing $5 \% \mathrm{HNO}_{3}$ ). 
According to the calibration curve, the SPION loading content (SLC) and SPION loading efficiency (SLE) can be determined.

The grafting content of FA on the surface of SPION-AN was quantified by using a UV-vis spectrophotometer (Lambda 950, PerkinElmer, USA). Typically, after preparation and centrifugation of SPION-AN-FA, the un-reacted FA remaining in the supernatant was measured by using a UV-vis spectrophotometer. The absorbance (wavelength $=363 \mathrm{~nm}$ ) of the supernatant was converted to FA concentration by using a calibration curve constructed from standard FA solutions with different concentrations. A simple mass balance was then used to calculate the FA conjugation amount on the surface of SPION-AN.

2.5.2 Morphology and size distributions of the nanoparticles. The morphology of SPION, SPION-AN-FA, and SPIONAN-FA@mPEG was characterized by using transmission electron microscopy (TEM). TEM images were recorded using a JEOL-2100 (JEOL, Japan) instrument operated at $200 \mathrm{KV}$. The hydrodynamic size and size distributions of SPION-AN-FA and SPION-ANFA@mPEG were measured by dynamic light scattering (DLS) using a Zeta Particle Size Analyzer (Nano-ZS, Malvern, England).

2.5.3 Degradation of AN. $40 \mathrm{~mL}$ of $4.4 \mathrm{mg} \mathrm{mL}^{-1}$ AN solution at $\mathrm{pH}$ 7.4, 6.5 and 5.5 was incubated at $37{ }^{\circ} \mathrm{C}$, respectively. At predetermined time intervals, $2.0 \mathrm{~mL}$ of the sample solutions was taken out and centrifuged $(18000 \mathrm{~g}, 10 \mathrm{~min})$. The harvested samples were then dried at $60{ }^{\circ} \mathrm{C}$ and the mass was calculated using a balance.

2.5.4 Magnetic properties of the nanoparticles. The magnetic properties of SPION, SPION-AN-FA, and SPION-AN-FA@mPEG were characterized using a Quantum Design Model-9PPMS (Quantum Design, USA) by measuring the applied dependence of magnetization between -30 and $30 \mathrm{KOe}$ at $300 \mathrm{~K}$.

2.5.5 High performance liquid chromatography (HPLC) measurement. Hydrolysis of the mPEGA-DCA polymer was measured and calculated using high performance liquid chromatography (HPLC) with a 1260 HPLC instrument (Agilent, USA). Briefly, $13.0 \mathrm{~mL}$ of mPEGA-DCA solutions at $\mathrm{pH} 7.4$ or 5.5 was incubated at $37{ }^{\circ} \mathrm{C}$. At predetermined time intervals, $0.5 \mathrm{~mL}$ of the sample solutions was taken out and added into $1.0 \mathrm{~mL}$ of PBS ( $\mathrm{pH}=7.4)$ for the HPLC analysis. The determination was performed on an RP-C18 HPLC column $(250.0 \times$ $\left.460.0 \mathrm{~mm}^{2}, 5.0 \mu \mathrm{m}\right)$. The mobile phase for elution was 40:60 acetonitrile/water at a flow rate of $1.0 \mathrm{~mL} \mathrm{~min}^{-1}$ for $4 \mathrm{~min}$. The volume of each injection was $5.0 \mu \mathrm{L}$. The detection of the eluted sample was performed at $250.0 \mathrm{~nm}$. The obtained peak area of the sample was converted into DCA concentration by using a calibration curve constructed with standard DCA solutions.

2.5.6 Nuclear magnetic resonance spectroscopy analysis. The hydrolysis of mPEGA-DCA was also measured and calculated using nuclear magnetic resonance (NMR) spectroscopy. The measurement was performed by using a ${ }^{1} \mathrm{H}-\mathrm{NMR}$ spectrophotometer (AVANCE III $400 \mathrm{MHz}$, Bruker, USA) using an internal standard method. The preparation of the hydrolyzed samples was the same as that of the HPLC analysis. After freezedrying, the samples were dispersed in $0.5 \mathrm{~mL}$ of deuterium oxide $\left(\mathrm{D}_{2} \mathrm{O}, 99.8\right.$ atom\% $\left.\mathrm{D}\right)$ with following addition of dimethyl sulfoxide as the internal standard substance (same amount for each sample). The peak area of $-\mathrm{CH}_{2}-\mathrm{NH}_{2}$ was used to calculate the hydrolyzed amount of mPEGA-DCA.

2.5.7 MRI performance of the nanoparticles. The in vitro MRI performance of SPION and SPION-AN-FA@mPEG was studied by using a MRI instrument with a magnetic field of $0.5 \mathrm{~T}$ (MesoMR23-060H-1, Niumag, China). The samples with various Fe concentrations (0.025-1.6 mM) were prepared to measure their longitudinal relaxivity $\left(r_{1}\right)$ and transverse relaxivity $\left(r_{2}\right)$.

\section{Results and discussion}

\subsection{Synthesis and characterization of SPION-AN-FA nanoparticles}

SPION was synthesized by a polyol method, and then encapsulated into AN followed by FA conjugation to construct SPIONAN-FA nanoparticles (Fig. 2a). TEM images in Fig. 3a and b confirm that the shapes of SPION and SPION-AN-FA nanoparticles are spherical, their sizes are uniform, and SPION was successfully encapsulated in AN. The particle sizes measured from the TEM images are 19 and $147 \mathrm{~nm}$ for SPION and SPIO-AN-FA, respectively. The amount of FA conjugated on the surface of SPION-AN-FA nanoparticles, i.e. mass percentage of FA with respect to SPION-AN-FA, was determined to be $3.03 \%$. SPION loading content (SLC) of SPION-AN-FA, i.e. mass percentage of SPION with respect to SPION-AN-FA, was measured to be $4.7 \%$.

\subsection{Synthesis and characterization of mPEG-DCA}

The pH-sensitive polymer (mPEG-DCA) was synthesized according to a reported method. ${ }^{27}$ Briefly, the reaction between $-\mathrm{NH}_{2}$ of mPEG and anhydride of DCA at pH 8.0-9.0 was utilized to synthesize mPEG-DCA (Fig. 2b).
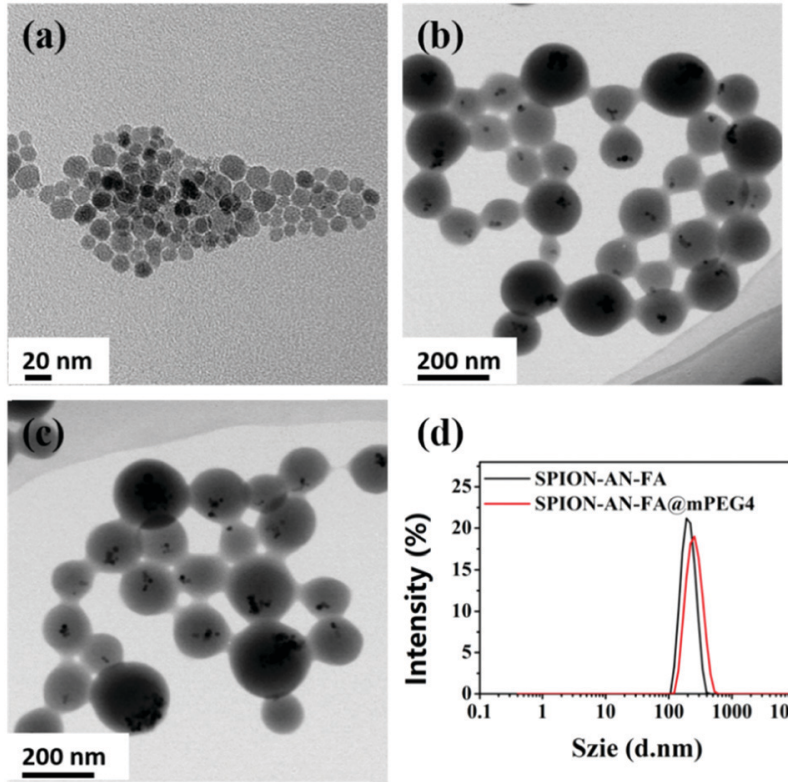

(d)

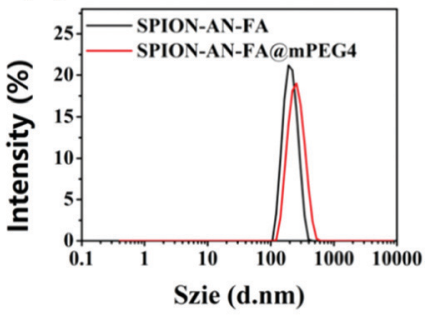

Fig. $3(\mathrm{a}-\mathrm{c})$ TEM images of the SPION, SPION-AN-FA, or SPION-ANFA@mPEG4 nanoparticles; (d) size distributions of SPION-AN-FA or SPION-AN-FA@mPEG4 nanoparticles measured by DLS. 
In order to evaluate the pH-sensitivity of mPEG-DCA, high performance liquid chromatography (HPLC) characterization was conducted. Various concentrations of DCA (as the standard substance) were first measured by HPLC to construct a standard curve (Fig. S1, ESI $\dagger$ ), which can be used for the quantitative analysis of DCA. Fig. 4 shows the characterization of mPEGA-DCA hydrolysis at $\mathrm{pH} 7.4$ or $\mathrm{pH} 5.5$ measured by using HPLC. It was found that the peak area of mPEGA-DCA incubated at pH 5.5 decreased obviously with increasing incubation time (Fig. 4a), which indicates that mPEG-DCA hydrolyzed rapidly in an acidic environment. In contrast, mPEG-DCA hydrolyzes very slowly at pH 7.4 (Fig. 4b).

For further verification of the pH-sensitivity of mPEG-DCA, ${ }^{1} \mathrm{H}$-NMR spectroscopy was also conducted. Fig. 5 shows the characterization of mPEGA-DCA hydrolysis at $\mathrm{pH} 7.4$ or pH 5.5 measured by a ${ }^{1} \mathrm{H}-\mathrm{NMR}$ spectrophotometer. In the ${ }^{1} \mathrm{H}-\mathrm{NMR}$ spectra, the peak area (at $3.12 \mathrm{ppm}$ ) of the $\mathrm{N}-\mathrm{H}$ bond of the hydrolyzed mPEGA-DCA at $\mathrm{pH} 7.4$ is almost the same at different time points (Fig. 5a), whereas that of the hydrolyzed mPEGA-DCA at pH 5.5 increased obviously with increasing incubation time (Fig. 5b). The hydrolysis behavior of mPEGADCA measured by ${ }^{1} \mathrm{H}-\mathrm{NMR}$ spectroscopy looks similar to that determined by HPLC (Fig. 6). From HPLC and NMR analysis, the hydrolysis ratio respectively reaches $60.62 \%$ and $85.65 \%$ after $5 \mathrm{~h}$ of incubation at $\mathrm{pH}$ 5.5. However, it is respectively only $23.96 \%$ and $17.9 \%$ even after $72 \mathrm{~h}$ of incubation at $\mathrm{pH} 7.4$.

\subsection{Optimization of SPION-AN-FA@MPEG synthesis}

In this study, in order to realize the precise tumor targeting of nanoparticles with reduced uptake by non-cancerous cells,

(a)

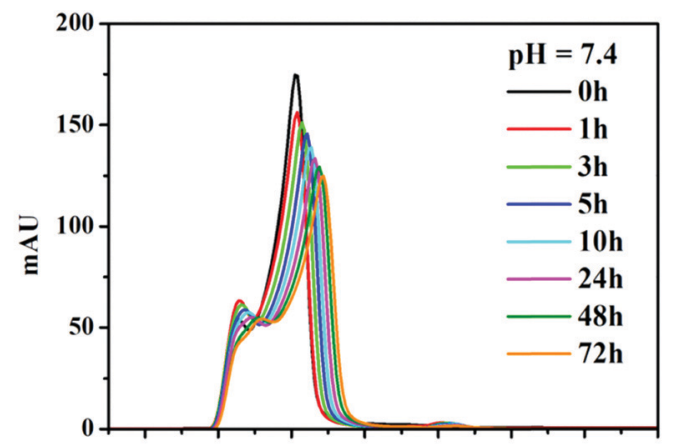

(b)

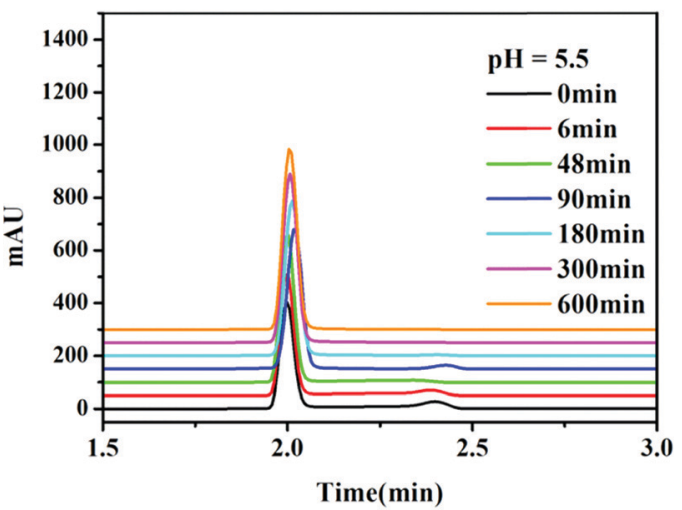

Fig. 4 Characterization of mPEGA-DCA hydrolysis at $\mathrm{pH} 7.4$ (a) or $\mathrm{pH} 5.5$ (b) measured by HPLC.

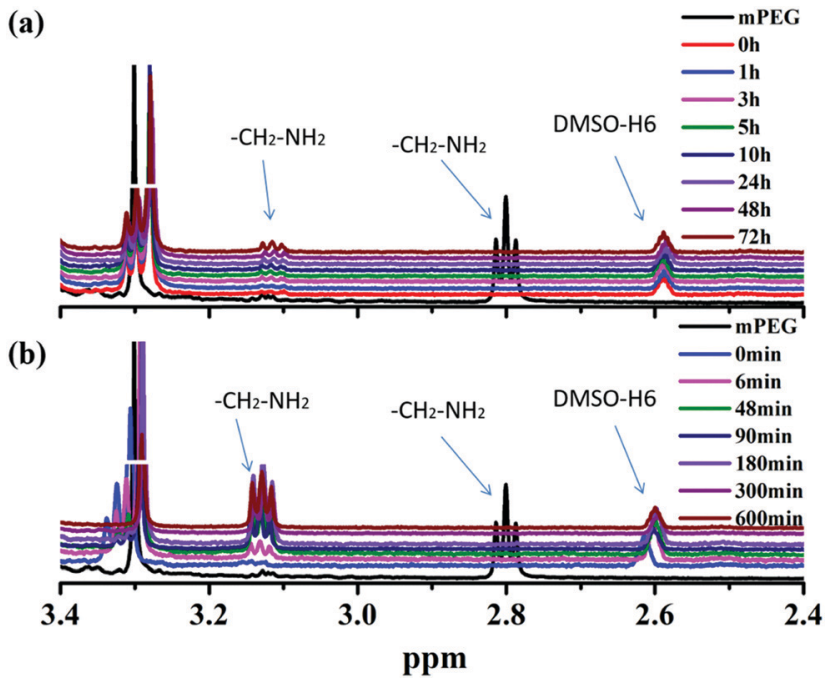

Fig. 5 Characterization of mPEGA-DCA hydrolysis at $\mathrm{pH} 7.4$ (a) or $\mathrm{pH} 5.5$ (b) measured by ${ }^{1} \mathrm{H}-\mathrm{NMR}$ spectroscopy.

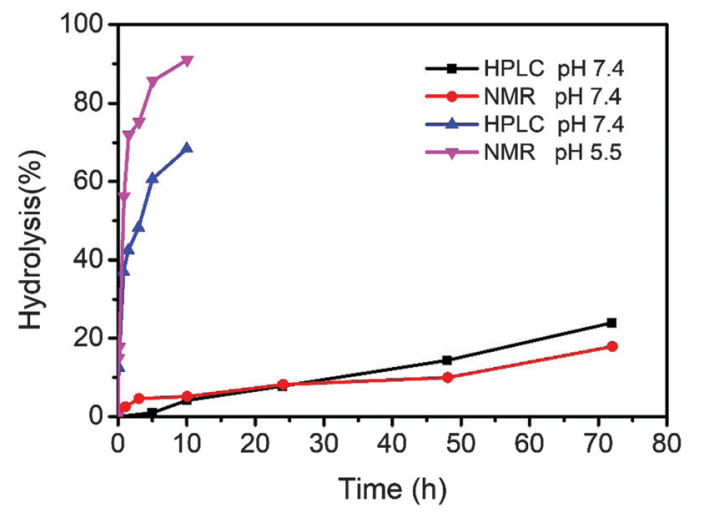

Fig. 6 The hydrolysis rate of mPEGA-DCA at pH 7.4 or 5.5 measured by HPLC or ${ }^{1} \mathrm{H}-\mathrm{NMR}$ spectroscopy.

the pH-sensitive polymer mPEG-DCA was conjugated onto the surface of SPION-AN-FA (Fig. 2c) to shield FA, avoiding non-specific recognition by normal cells under physiological conditions, and to shed to expose FA in tumor microenvironments. Therefore, the graft density and length of mPEG-DCA are the key parameters to realize the precise tumor targeting of SPION-ANFA@mPEG.

Table S1 (ESI $\dagger$ ) shows the synthesis conditions of SPIONAN-FA@mPEG1-6 nanoparticles with various graft density and length of mPEG-DCA. Fig. 7a-d shows the flow cytometry analysis of MCF-7 cells without or with incubation of SPIONAN-FA@mPEG1-6 nanoparticles, which were pre-incubated at pH 5.5 or 7.4 in PBS for $12 \mathrm{~h}$ before incubation with cells. The AN nanoparticles exhibit a strong green autofluorescence (FITC-like). ${ }^{13}$ It was found that the fluorescence intensity of MCF-7 cells with incubation of SPION-AN-FA@mPEG1-6 nanoparticles pre-incubated at $\mathrm{pH} 5.5$ in PBS is much stronger than that of the pre-incubated samples at $\mathrm{pH}$ 7.4. This result demonstrates that the $\mathrm{pH}$-sensitive polymer mPEG-DCA can be shed from SPION-AN-FA@mPEG1-6 nanoparticles at pH 5.5 to 
(a)

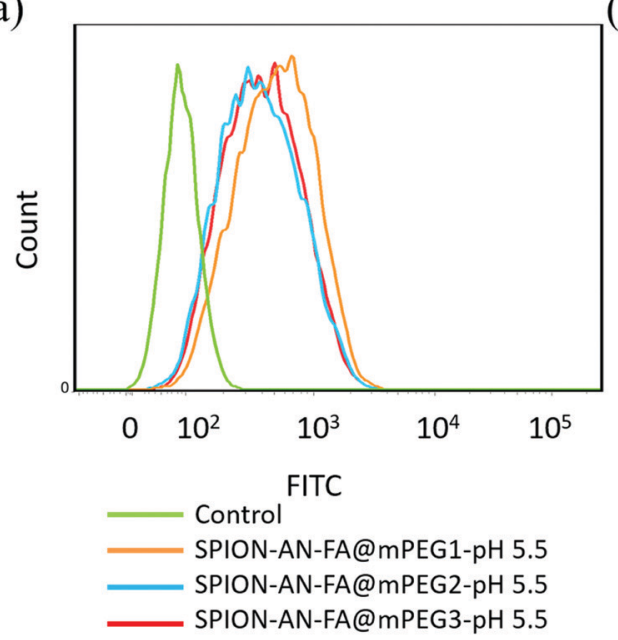

(c)

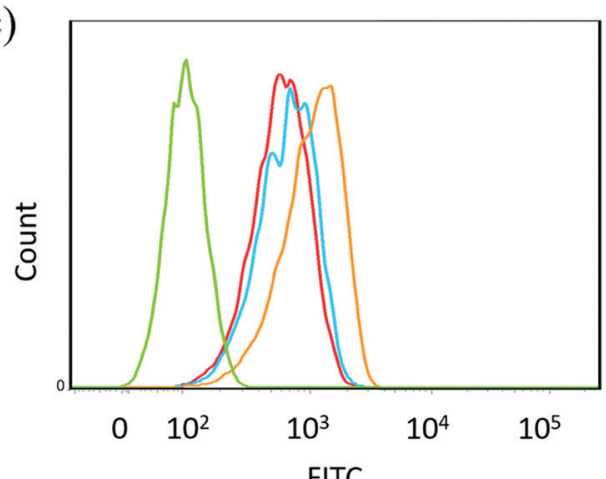

FITC

Control

SPION-AN-FA@mPEG4-pH 5.5

SPION-AN-FA@mPEG5-pH 5.5

SPION-AN-FA@mPEG6-pH 5.5

(e)

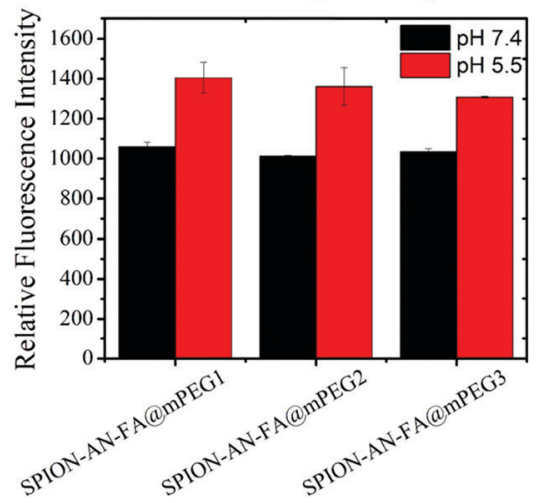

molecular weight of $\mathrm{mPEG}$ (b)

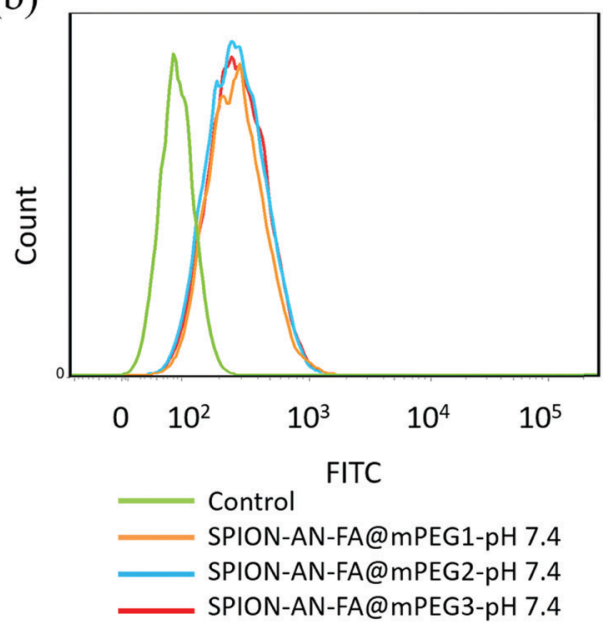

(d)
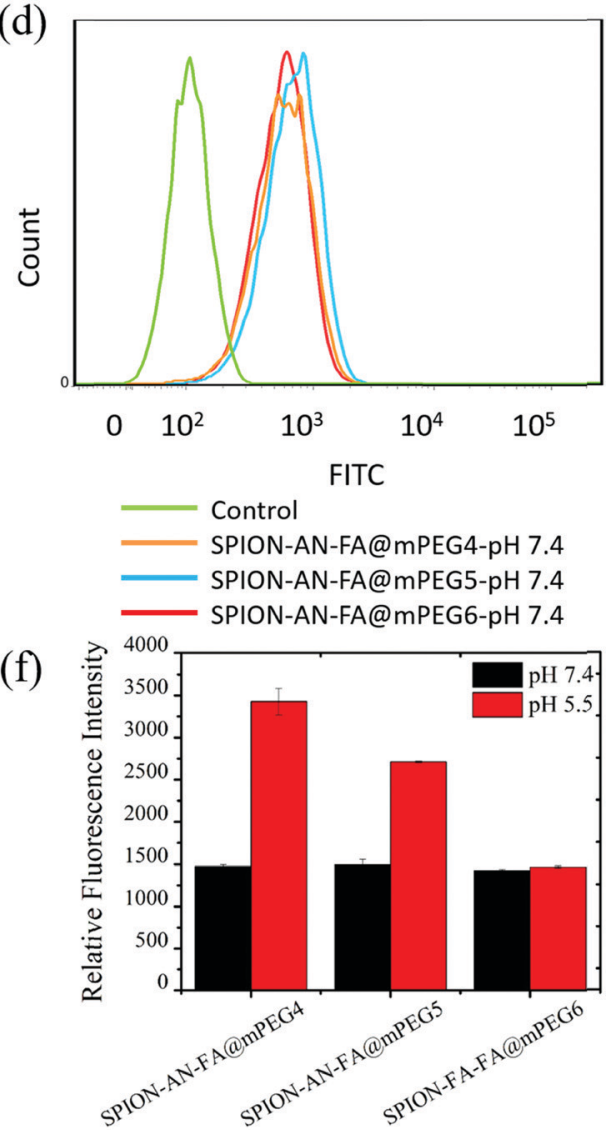

Feed ratio of mPEG to SPION-AN-FA

Fig. 7 Flow cytometry analysis of MCF-7 cells without (control) or with incubation of SPION-AN-FA@mPEG1-6 nanoparticles, which were pre-incubated at pH 5.5 ( $a$ and $c$ ) or 7.4 ( $b$ and d) in PBS for $12 \mathrm{~h}$ before incubation with cells. (a and $b$ ) The mPEG molecular weight is respectively 2, 5 or $10 \mathrm{kDa}$ for SPION-AN-FA@mPEG1-3 nanoparticles. (c and d) The mass ratio of mPEG to SPION-AN-FA is respectively $0.5,1$ or 2 for SPION-ANFA@mPEG4-6 nanoparticles. (e and f) The ratio of relative fluorescence intensity of MCF-7 cells treated with SPION-AN-FA@mPEG1-6 nanoparticles to untreated cells (i.e., relative fluorescence intensity).

expose FA, increasing the cellular uptake, but this cannot occur at $\mathrm{pH}$ 7.4. Therefore, our $\mathrm{pH}$-sensitive polymer based precise tumor targeting strategy is applicable to reduce the uptake of nanoparticles by non-cancerous cells.
To optimize the graft density and length of mPEG-DCA on SPION-AN-FA@MPEG nanoparticles, the differences in the relative fluorescence intensity of MCF-7 cells with incubation of SPION-AN-FA@mPEG1-6 pre-incubated at pH 5.5 and pH 7.4 
in PBS were calculated and are shown in Fig. 7e and f. Due to the higher difference between $\mathrm{pH} 5.5$ and $\mathrm{pH} 7.4$ of PBS pre-incubation, the molecular weight of mPEG (i.e., graft length) was optimized to be $2 \mathrm{kDa}$, and the mass ratio of MPEG to SPION-AN-FA (i.e., graft density) was optimized to be 0.5 . Therefore, SPION-AN-FA@mPEG4 has an optimal graft density and length of mPEG-DCA to realize the precise tumor targeting strategy.

The SPION-AN-FA@mPEG4 nanoparticle dispersions were purified by washing with Milli-Q water and collection using a neodymium magnet. To determine the nanoparticle purity, $3.0 \mathrm{~mL}$ of SPION-AN-FA@mPEG4 nanoparticle dispersion and that after centrifugation $(20000 \mathrm{~g}, 30 \mathrm{~min})$ were dried at $70{ }^{\circ} \mathrm{C}$ to measure the mass difference. The purity of SPION-ANFA@mPEG4 was measured to be $81 \%$.

To further demonstrate that mPEG-DCA could fall off from the surface of SPION-AN-FA under acidic conditions, the relative fluorescence intensity of MCF-7 cells with incubation of SPION-AN-FA@MPEG4 nanoparticles, which were pretreated at pH 7.4, 6.5 and 5.5 in PBS for $12 \mathrm{~h}$, was measured by flow cytometry analysis and the result is shown in Fig. S2 (ESI $\dagger$ ). It was found that the relative fluorescence intensities of the cases of $\mathrm{pH} 6.5$ and 5.5 are both higher than that of $\mathrm{pH}$ 7.4. Therefore, our nanoparticles are responsive to the acidic condition of the tumor microenvironment and can realize the precise tumor targeting strategy.

\subsection{Degradation of albumin nanoparticles}

The degradation ratio of $\mathrm{AN}$ was measured at $\mathrm{pH} 7.4,6.5$ and 5.5, as shown in Fig. S3 (ESI $\dagger$ ). The degradation of AN at $\mathrm{pH} 7.4$ is almost negligible, but it is faster at $\mathrm{pH} 6.5$ and 5.5. After 4 days of incubation, $5.9 \%$ or $9.3 \%$ AN was degraded at $\mathrm{pH} 6.5$ or $\mathrm{pH}$ 5.5. Therefore, nanocarrier degradation at acidic $\mathrm{pH}$ is higher than that under physiological conditions. These results indicate that our SPION-AN-FA can be stable in the blood circulation and can be used for targeted drug release in the tumor site.

\subsection{Characterization of the optimized SPION-AN-FA@mPEG}

Fig. 3c shows the TEM image of SPION-AN-FA@mPEG4 nanoparticles, whose sizes and morphologies are similar to that of SPION-AN-FA nanoparticles (Fig. 3b) because the polymer
mPEG on the nanoparticle surface is not visible. Fig. 3d shows the size distributions of SPION-AN-FA and SPION-AN-FA@mPEG4 nanoparticles measured by DLS. Compared with SPION-AN-FA, SPION-AN-FA@mPEG4 has a narrow size distribution due to the hydrophilic polymer mPEG on the surface. In addition, the hydrodynamic particle size of SPION-AN-FA@mPEG4 was measured to be $190 \mathrm{~nm}$, which is slightly larger than that of SPION-AN-FA $(179 \mathrm{~nm})$. The $\mathrm{M}-\mathrm{H}$ curve of SPION-ANFA@mPEG4 was also characterized by a physical property measurement system (PPMS) (Fig. S4, ESI $\dagger$ ). Its Ms value was measured to be $19.8 \mathrm{emu} \mathrm{g}^{-1}$, which is much smaller than that of SPION (110 emu $\mathrm{g}^{-1}$ ) due to the non-magnetic components of AN, FA and mPEG.

\subsection{In vitro MRI studies of the optimized SPION-AN- FA@MPEG}

Fig. 8a-c show the $T_{1}$ or $T_{2}$ relaxation rate $(1 / T)$ as a function of Fe concentration at $0.5 \mathrm{~T}$ for SPION, SPION-AN-FA, and SPIONAN-FA@mPEG4, respectively. Both longitudinal relaxivity $\left(r_{1}\right)$ and transverse relaxivity $\left(r_{2}\right)$ of the nanoparticles were calculated from the slopes of the linear relationships, as shown in Fig. 8a-c. The $r_{2}$ value of the SPION, SPION-AN-FA, and SPION-AN-FA@mPEG samples was respectively determined to be $100.2,165.2$, and $168.6 \mathrm{mM}^{-1} \mathrm{~s}^{-1}$. The $r_{2} / r_{1}$ ratio of the SPION, SPION-AN-FA, and SPION-AN-FA@mPEG samples was calculated to be $8.3,43.1$, and 42.8 , respectively. Both the $r_{2}$ value and $r_{2} / r_{1}$ ratio of SPION-AN-FA and SPION-AN-FA@mPEG are much higher than that of SPION. This is because SPION is embedded in SPION-AN-FA and SPION-AN-FA@mPEG in an aggregation state, resulting in a bigger radius of the magnetic cores, which thus leads to the higher $r_{2}$ values of SPION-AN-FA and SPION-AN-FA@mPEG according to the following equation. ${ }^{28}$ In eqn (1), $r$ is the radius of the magnetic core.

$$
\frac{1}{T_{2}}=\frac{\left(256 \pi^{2} \gamma^{2} / 405\right) V^{*} M_{\mathrm{s}}^{2} r^{2}}{D(1+L / r)}
$$

The $r_{2}$ value and $r_{2} / r_{1}$ ratio of SPION-AN-FA@mPEG4 are both much higher than that of the commercial contrast agent Resovist $^{\mathbb{R}}\left(r_{2}=82.0 \mathrm{mM}^{-1} \mathrm{~s}^{-1}, r_{2} / r_{1}=11.4\right),{ }^{29}$ which demonstrates that our SPION-AN-FA@mPEG4 has a stronger $T_{2}$ shortening effect and is feasible to be used as a better $T_{2}$-weighted MRI contrast agent.

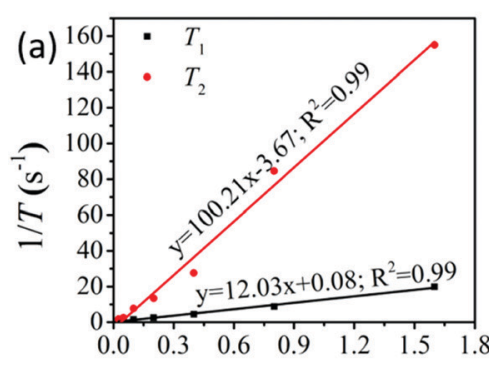

Concentration of $\mathrm{Fe}(\mathrm{mM})$
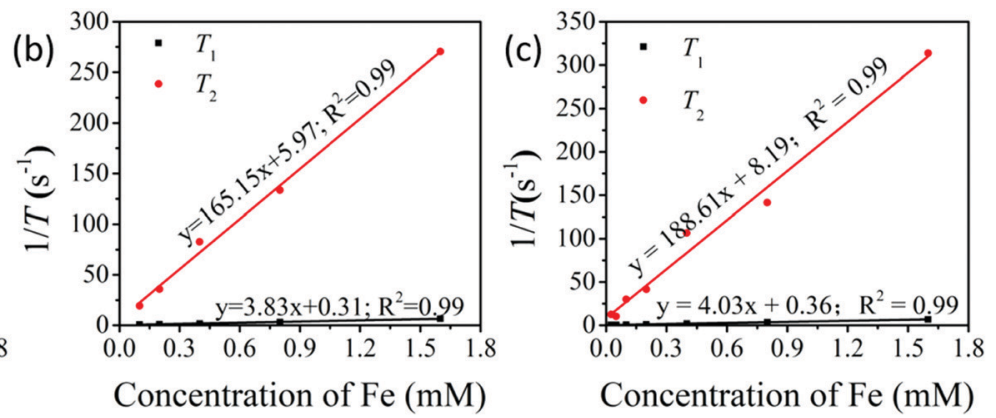

Fig. 8 Relaxation rate $\left(1 / T, \mathrm{~s}^{-1}\right)$ plotted as a function of Fe concentration (mM) for (a) SPION, (b) SPION-AN-FA and (c) SPION-AN-FA@mPEG4. Magnetic field $=0.5 \mathrm{~T}$. 
The $T_{2}$-weighted MR images of the SPION, SPION-AN-FA, and SPION-AN-FA@mPEG4 nanoparticles with different Fe concentrations are shown in Fig. S5a-c (ESI $\dagger)$. Fig. S5d-f (ESI $\dagger$ ) show the corresponding pseudo color pictures. It was found that the brightness of the $T_{2}$-weighted MR images decreases significantly with increasing Fe concentration, indicating that SPION, SPION-AN-FA, and SPION-AN-FA@mPEG4 all have a good MRI contrast effect under the $T_{2}$-weighted sequence and can be used as negative MRI contrast agents. In addition, compared with SPION at the same Fe concentrations, SPIONAN-FA and SPION-AN-FA@mPEG generate much darker images, which can be attributed to the state of the iron oxide in AN and the higher $r_{2}$ and $r_{2} / r_{1}$. The differences between the image brightness of SPION-AN-FA and that of SPION-ANFA@MPEG at the same Fe concentrations are negligible, which indicates that the MPEG conjugation has a minor influence on the $T_{2}$-weighted MR imaging. Because of the excellent waterdispersibility $(\mathrm{PDI}=0.064)$ and the strong $T_{2}$ imaging efficiency, our SPION-AN-FA@mPEG4 nanoparticles can be used as an effective $T_{2}$-weighted MRI contrast agent.

\subsection{In vivo MRI performance of the optimized SPION-AN- FA@MPEG}

MCF-7 tumor bearing nude mice were intravenously injected with SPION, SPION-AN-FA or SPION-AN-FA@mPEG4 nanoparticles to investigate the in vivo MRI performance. Fig. S6 (ESI $\dagger$ ) shows the $T_{2}$-weighted MR images and corresponding pseudo color pictures of tumor-bearing mice pre- or postinjection of SPION, SPION-AN-FA, or SPION-AN-FA@mPEG4 nanoparticles. It can be seen that the MRI signal intensity of the tumor site decreases gradually after intravenous injection of SPION, SPION-AN-FA, or SPION-AN-FA@mPEG4 nanoparticles because they are all negative MRI contrast agents. From the MR images, we can see the MRI signal at the tumor site is darkest at $4 \mathrm{~h}$ post-injection (i.v.) of SPION nanoparticles, whereas it is darkest at $24 \mathrm{~h}$ post-injection of SPION-AN-FA or SPION-AN-FA@mPEG4 nanoparticles. This is because SPION-AN-FA and SPION-AN-FA@mPEG4 have a longer blood circulation time than SPION due to the hydrophilicity of AN. In addition, it was found that SPION-AN-FA@mPEG4 has a better tumor darkening efficiency compared to the control (pre-injection) than SPION-AN-FA. This result demonstrates that the $\mathrm{pH}$-sensitive polymer $\mathrm{MPEG}$ can reduce the nonspecific uptake of SPION-AN-FA@mPEG4 nanoparticles by non-cancerous cells, and enhance the tumor accumulation of the nanoparticles.

\subsection{In vitro studies of nanoparticles}

In order to demonstrate that the nanoparticles can be more easily uptaken by tumor cells, the cellular uptake of SPION-ANFA@mPEG4 by MCF-7 cells was tested by using laser scanning confocal microscopy (LSCM). Fig. S7 (ESI $\dagger$ ) shows the LSCM images of the MCF-7 cells incubated with SPION-AN-FA@ mPEG4, which was pretreated at $\mathrm{pH} 5.5$ or 7.4 in PBS for $12 \mathrm{~h}$ at $37{ }^{\circ} \mathrm{C}$. The MCF-7 cells without incubation of nanoparticles were used as a control. The samples were simultaneously excited at 405 and $488 \mathrm{~nm}$. The nuclei stained with Hoechst (EM 420-480 nm) are blue at an excitation of $405 \mathrm{~nm}$. SPIONAN-FA@mPEG4 (EM 510-540) is green under excitation at $488 \mathrm{~nm}$. It was found that more SPION-AN-FA@mPEG4 nanoparticles pretreated at $\mathrm{pH} 5.5$ were internalized in the MCF-7 cells than that pretreated at $\mathrm{pH}$ 7.4. The results indicate that the nanoparticles can be more easily uptaken by tumor cells.

\subsection{In vitro and in vivo toxicity evaluation}

In vitro cytotoxicity of the optimized SPION-AN-FA@mPEG4 nanoparticles was first evaluated by the MTT assay on MCF-7 cells. Fig. S8 (ESI $\dagger$ ) shows the cell viability of MCF-7 cells incubated with SPION, SPION-AN-FA or SPION-AN-FA@mPEG at different iron concentrations. The cell viability is $\sim 95 \%$ for cells incubated with SPION at $0.1 \mathrm{mM}$ iron concentration, and it decreases with increasing Fe concentration. However, the cell viability is higher than $80 \%$ at any $\mathrm{Fe}$ concentration, which indicates that the SPION nanoparticles have a good biocompatibility. Compared with SPION, SPION-AN-FA and SPION-AN-FA@mPEG4 nanoparticles show higher cell viabilities at most of the Fe concentrations. In addition, compared with SPION and SPION-AN-FA, SPION-AN-FA@mPEG4 nanoparticles show lower cytotoxicity at any Fe concentration, which further indicates that MPEG could effectively hide the FA molecules on SPION-AN-FA@mPEG4 nanoparticles and reduce the non-specific uptake by cells at $\mathrm{pH}$ 7.4.

Furthermore, we carried out the pathological analysis of mouse main organs to further evaluate the biocompatibility of SPION-AN-FA@mPEG4 nanoparticles (Fig. S9, ESI $\dagger$ ). Histological analysis shows that the heart, liver, spleen, lung and kidney of the mice post-injection of SPION-AN-FA@mPEG4 nanoparticles are very similar to the control (i.e., post-injection of PBS). These results reinforce that our SPION-AN-FA@mPEG4 nanoparticles are biocompatible.

\section{Conclusions}

In summary, we proposed a $\mathrm{pH}$-sensitive polymer based precise tumor targeting strategy to reduce the uptake of nanoparticles by non-cancerous cells, and synthesized SPION-encapsulated AN with conjugation of FA and MPEG-DCA (SPION-AN-FA@MPEG). From the HPLC and NMR analysis, the hydrolysis ratio of mPEG-DCA respectively reaches $60.62 \%$ and $85.65 \%$ after $5 \mathrm{~h}$ of incubation at $\mathrm{pH} 5.5$. However, it is respectively only $23.96 \%$ and $17.9 \%$ even after $72 \mathrm{~h}$ of incubation at $\mathrm{pH}$ 7.4. The graft density and length of mPEG-DCA were optimized via the cellular uptake of SPION-AN-FA@mPEG measured by using flow cytometry. The $r_{2}$ value and $r_{2} / r_{1}$ ratio of the optimized SPION-AN-FA@mPEG (i.e., SPION-AN-FA@mPEG4) are respectively $168.6 \mathrm{mM}^{-1} \mathrm{~s}^{-1}$ and 42.8 , which are both much higher than that of the commercial contrast agent Resovist ${ }^{\mathbb{R}}$. The in vitro $T_{2}$-weighted $\mathrm{MR}$ images and in vivo MRI performance indicate that our SPION-AN-FA@mPEG4 has a stronger $T_{2}$ shortening effect and can be used as an effective $T_{2}$-weighted MRI contrast agent. In vitro and in vivo toxicity evaluations 
reinforced that our SPION-AN-FA@mPEG4 nanoparticles are biocompatible.

\section{Conflicts of interest}

There are no conflicts to declare.

\section{Acknowledgements}

This work was supported in part by the Zhejiang Provincial Natural Science Foundation of China (R19E030002, LQ19H180002), Youth Innovation Promotion Association of the Chinese Academy of Sciences (2016269) (Z. S.), National Natural Science Foundation of China (Grant No. 51761145021 (BRICS Research Project), 51602203, U1501501, and U1432114), Zhejiang Province Public Welfare Technology Application Research Project (2017C33129), National Key Research \& Development Program (Grant No. 2016YFC1400600, 2018YFD0800300, and 2018YFC0910601), and Key Program of Zhejiang Province Medical Scientific Research Foundation (Grant No. WKJ-ZJ-1807). Aiguo Wu thanks Hundred Talents Program of Chinese Academy of Sciences (2010-735).

\section{References}

1 M. W. Dewhirst and D. Kirsch, JAMA Oncol., 2018, 4, 493-494. 2 M. Maemondo, A. Inoue, K. Kobayashi, S. Sugawara, S. Oizumi, H. Isobe, A. Gemma, M. Harada, H. Yoshizawa, I. Kinoshita, Y. Fujita, S. Okinaga, H. Hirano, K. Yoshimori, T. Harada, T. Ogura, M. Ando, H. Miyazawa, T. Tanaka, Y. Saijo, K. Hagiwara, S. Morita and T. Nukiwa, N. Engl. J. Med., 2010, 362, 2380-2388.

3 J. P. Gerard, P. Romestaing and O. Chapet, Lancet Oncol., 2003, 4, 158-166.

4 J. Ruan, Y. Wang, F. Li, R. B. Jia, G. G. Zhou, C. L. Shao, L. Q. Zhu, M. L. Cui., D. P. Yang and S. F. Ge, ACS Appl. Mater. Interfaces, 2018, 10, 14342-14355.

5 J. C. Coffey, J. H. Wang, M. J. F. Smith, D. Bouchier-Hayes and H. P. Redmond, Lancet Oncol., 2003, 4, 760-768.

6 D. Q. Li, W. Jiang, J. S. Bishop, R. Ralston and B. W. O’Malley, Clin. Cancer Res., 1999, 5, 1551-1556.

7 J. D. Seitz, J. G. Vineberg, E. Herlihy, B. Park, E. Melief and I. Ojima, Bioorg. Med. Chem., 2015, 23, 2187-2194.

8 L. Bello, G. Carrabba, C. Giussani, V. Lucini, F. Cerutti, F. Scaglione, J. Landre, M. Pluderi, G. Tomei, R. Villani, R. S. Carroll, P. M. Black and A. Bikfalvi, Cancer Res., 2001, 61, 7501-7506.

9 S. Sultana, M. R. Khan, M. Kumar, S. Kumar and M. Ali, J. Drug Targeting, 2012, 21, 107-125.

10 Y. Tsvetkova, N. Beztsinna, M. Baues, D. Klein, A. Rix, S. K. Golombek, W. A. Rawashdeh, F. Gremse, M. Barz, K. Koynov, S. Banala, W. Lederle, T. Lammers and F. Kiessling, Nano Lett., 2017, 17, 4665-4674.
11 P. Moitra, K. Kumar, S. Sarkar, P. Kondaiah, W. Duand and S. Bhattacharya, Chem. Commun., 2017, 53, 8184-8187.

12 C. Y. Zhang, D. Wu, L. J. Lu, X. H. Duan, J. Liu, X. Y. Xie, X. T. Shuai, J. Shen and Z. Cao, ACS Biomater. Sci. Eng., 2018, 4, 2597-2605.

13 X. H. Ma, A. Gong, L. C. Xiang, T. X. Chen, Y. X. Gao, X. J. Liang, Z. Y. Shen and A. G. Wu, J. Mater. Chem. B, 2013, 1, 3419-3428.

14 Y. Z. Xia, X. X. Wu, J. T. Zhao, J. S. Zhao, Z. H. Li, W. Z. Ren, Y. C. Tian, A. G. Li, Z. Y. Shen and A. G. Wu, Nanoscale, 2016, 8, 18682-18692.

15 G. Wu, D. Kim, J. N. Kim, S. Park, S. Maharjan, H. Koh, K. Moon, Y. Lee and H. J. Kwon, Theranostics, 2018, 8, 78-91.

16 Y. X. Ye, M. C. Shin, Q. L. Liang, H. N. He and V. C. Yang, J. Controlled Release, 2015, 205, 58-69.

17 Q. Dai, Y. Yan, C. S. Ang, K. Kempe, M. M. J. Kamphuis, S. J. Dodds and F. Caruso, ACS Nano, 2015, 9, 2876-2885.

18 V. P. Torchilin, A. N. Lukyanov, Z. G. Gao and B. P. Sternberg, PNAS, 2003, 100, 6039-6044.

19 Y. Sakurai, W. Mizumura, M. Murata, T. Hada, S. Yamamoto, K. Ito, K. Iwasaki, T. Katoh, Y. Goto, A. Takagi, M. Kohara, H. Suga and H. Harashima, Mol. Pharmaceutics, 2017, 14, 3290-3298.

20 M. Ying, Q. Shen, C. Y. Zhan, X. L. Wei, J. Gao, C. Xie, B. X. Yao and W. Y. Lu, J. Controlled Release, 2016, 243, 86-98.

21 Z. Wu, L. J. Tang, X. B. Zhang, J. H. Jiang and W. H. Tan, ACS Nano, 2011, 5, 7696-7699.

22 H. R. Liu, J. H. Mai, J. L. Shen, J. Wolfram, Z. Q. Li, G. D. Zhang, R. Xu, Y. Li, C. F. Mu, Y. L. Zu, X. Li, G. L. Lokesh, V. Thiviyanathan, D. E. Volk, D. G. Gorenstein, M. Ferrari, Z. B. Hu and H. F. Shen, Theranostics, 2018, 8, 31-44.

23 Z. Ye, W. R. Wu, Y. F. Qin, J. Hu, C. Liu, P. H. Seeberger and J. Yin, Adv. Funct. Mater., 2018, 28, 1706600.

24 Q. Zhang, S. N. Deng, J. L. Liu, X. X. Zhong, J. He, X. F. Chen, B. W. Feng, Y. F. Chen and K. K. Ostrikov, Adv. Funct. Mater., 2019, 29, 180586.

25 Z. Y. Shen, H. Wu, S. Yang, X. H. Ma, Z. H. Li, M. Q. Tan and A. G. Wu, Biomaterials, 2015, 70, 1-11.

26 X. H. Ma, A. Gong, B. Chen, J. J. Zheng, T. X. Chen, Z. Y. Shen and A. G. Wu, Colloids Surf., B, 2015, 126, 44-49.

27 J. Z. Du, X. J. Du, C. Q. Mao and J. Wang, J. Am. Chem. Soc., 2011, 133, 17560-17563.

28 Z. Y. Shen, T. X. Chen, X. H. Ma, W. Z. Ren, Z. J. Zhou, G. Z. Zhu, A. Zhang, Y. J. Liu, J. B. Song, Z. H. Li, H. M. Ruan, W. P. Fan, L. S. Lin, J. Munasinghe, X. Y. Chen and A. G. Wu, ACS Nano, 2017, 11, 10992-11004.

29 H. L. Ma, X. R. Qi, Y. Maitani and T. Nagai, Int. J. Pharm., 2007, 333, 177-186. 